\title{
Influence of low power consumption on IEEE 802.15.4 in wireless networks performance
}

\author{
A. Z. Yonis \\ Department of Communication Engineering, College of Electronics Engineering, Ninevah University, Mosul, Iraq
}

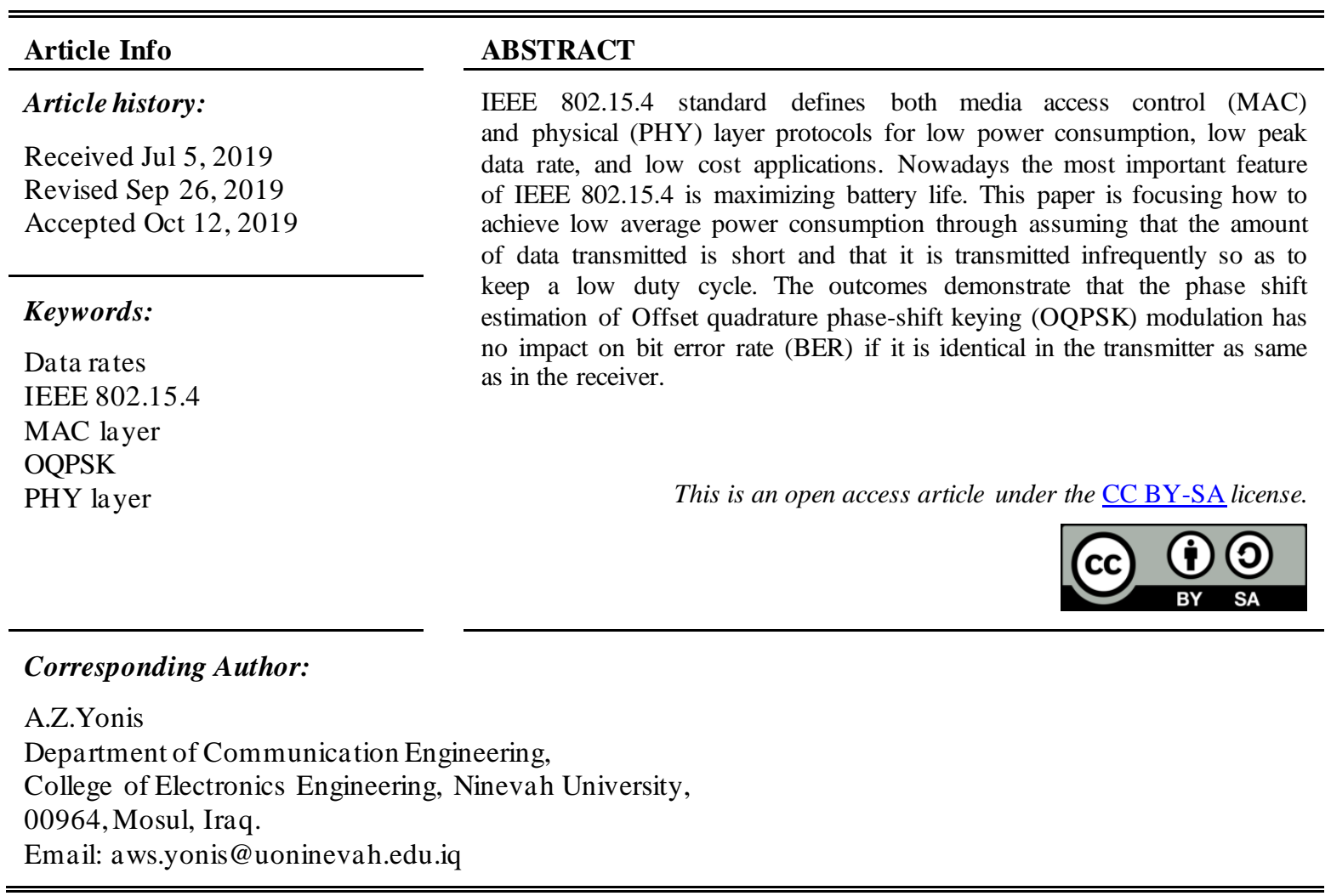

\section{INTRODUCTION}

IEEE 802.15.4 standard and it is the new technology that has been developed for low power consumption, low cost and low data rate wireless network. The reasons for using IEEE 802.15.4 technology are reliability, self-healing it is secure and can be used globally [1-2]. In the recent years, a new wireless network technology STD. The IEEE 802.15.4 [3-5], which is a standard built on the top of IEEE STD. It contain a suite of technologies that provide reliability, high security and can be used globally, also it is used to enable scalable that can manage various data traffic patterns. The experiments show the effects on bit error rate (BER) of the modulation process [6]. In addition, a comparison of BER values in terms of $\mathrm{Tx}$ and $\mathrm{Rx}$ parameters is done [7-8]. The organization of the research paper is as follows. Section two introduces the IEEE 802.15.4 channel structure. While, IEEE 802.15.4 transceiver system and configuration are illustrated in Section three. Then, simulation results and analysis study are described in Section four. Finally, Section five gives the conclusions of research paper.

\section{RELATED WORKS}

In fact, IEEE 802.15.4 system involves the physical and medium access control layers. They are used in order to support straightforward devices with minimum power work in POS (Personal Operating Space) of 10 meters as mentioned in [9]. On the other hand, in 802.15 .4 standard all the wireless connections are working in three frequency bands as shown in the following Figure 1. These bands allow free ISM (industrial scientific medical) with $250 \mathrm{kbps}$ peak data rates in 
band $2.4 \mathrm{GHz}, 40 \mathrm{kbps}$ in band $915 \mathrm{MHz}$ and finally $20 \mathrm{kbps}$ in band $868 \mathrm{MHz}$. The allocated channels in 802.15.4 are aggregated 27 channels. These channels are incorporating as the following: 16 channels through the band of $2.4 \mathrm{GHz}, 10$ channels through the band of $915 \mathrm{MHz}$ and the last channel in used in band $868 \mathrm{MHz}$. All the more explicitly, IEEE 802.15.4 includes layers top of this to include more system and application knowledge. IEEE 802.15.4 [10-11] is the reason for some other industrial wireless protocols also so understanding it can be extremely valuable to a security consultant [12]. The standard IEEE 802.15.4 is custom fitted to the requirements of sensor systems as shown in Figure 2. Therefore it permits more prominent extents at lower data rates and low power consumption [14].

\section{8/915 MHz PHY}
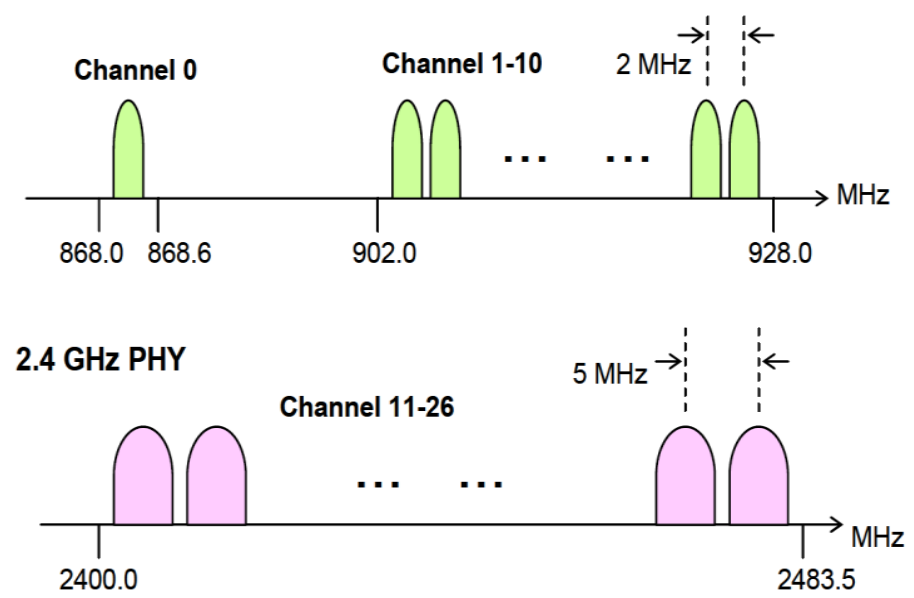

Figure 1. The structure of IEEE 802.15.4 channel[13]

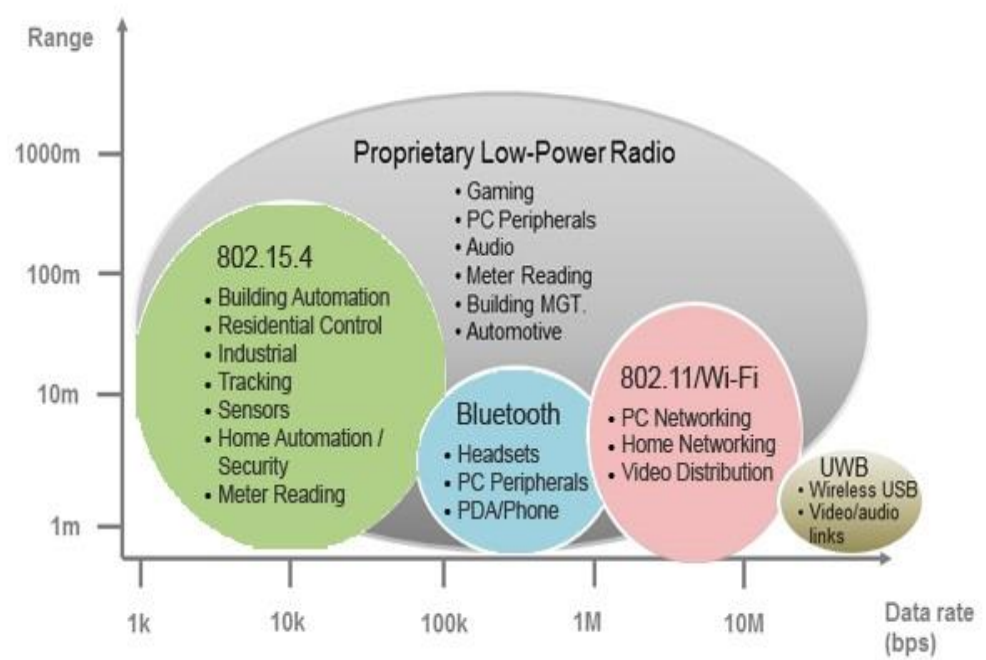

Figure 2. IEEE 802.15.4 and other relatedtechnologies

An IEEE 802.15.4 system enables a lot of devices to communicate wirelessly by means of one of a few potential topologies [15]. Packets of information can be sent between nodes and might be directed by intermediary devices to more distant nodes that would some way or a nother be out of range. Every device has both a MAC address and an IEEE 802.15.4 system address [16-17] and the system in general has its own personal area networks identifier shared by all devices. Packets can be ensured by encryption but for this to work all nodes will require a key. 


\section{IEEE 802.15.4 SYSTEM}

Addition of both in phase and quadrature signals after modulation, creates the required transmitter output. The required output signal is created by utilizing sum block in commonly used blocks. There will be no phase advances in the output, which is an advantageous property. This section of research paper, describes the implementation of IEEE 802.15.4 transmitter system. The design of IEEE 802.15.4 transmitter utilizing offset quadrature phase-shift keying (OQPSK) [18-19] modulation with half sine pulse shaping is appeared in the Figure 3.
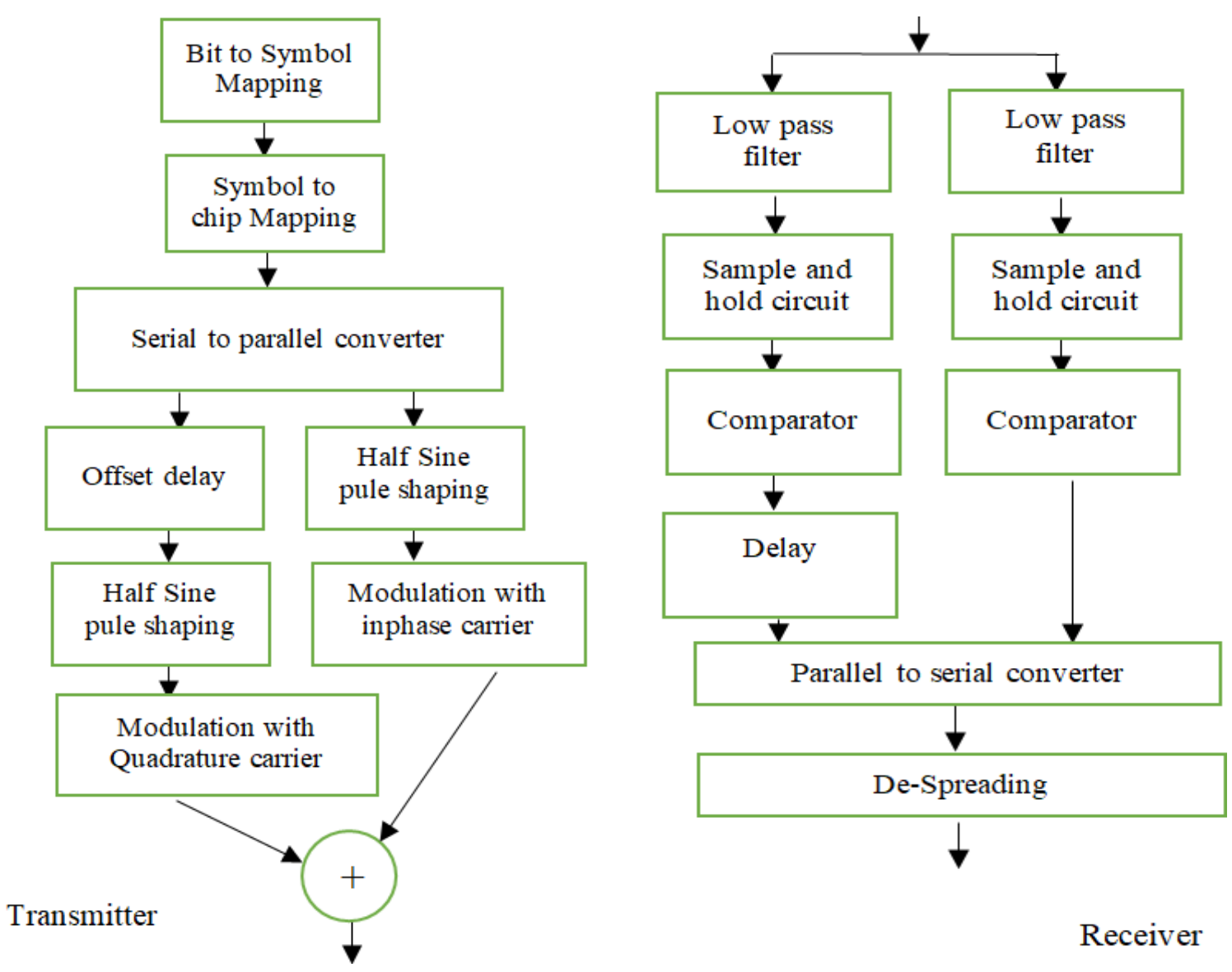

Figure 3.Transmitter and receiver of IEEE 802.15.4[20]

Here, the research describes the execution of IEEE 802.15.4 receiver system and we are focusing on the MSK coherent detection strategy for recovering unique information in the receiver, moreoverthe receiver of IEEE 802.15.4 has a ppeared in Figure 3.In the receiver configuration of IEEE 802.15.4, we are utilizing an MSK demodulator and a multiplier for dispreading[21]. This multiplier is provided by a PN sequence information that is an exact replica that utilized in the transmitter. The information originating from the MSK demodulator is having an information rate of two Mega bit per second alsofrom this information[22]. The original information is removed by increasing with the PN arrangement information. However, the $2 \mathrm{Mbps}$ information obtained at the output of parallel to sequential converter contains some offset delay. This offset delay must be presented in the PN sequence information while increasing with $2 \mathrm{Mbps}$ information, So that output contains unique bit stream without any errors.

The approaching received signal is connected to two synchronous demodulators, comprising of a multiplier followed by a low pass filter. Here one multiplier is provided with a signal, which is the duplicated signal of carrier coswot and $\cos (2 \pi \mathrm{t} / 4 \mathrm{~Tb})$. Another multiplier is provided with quadrature signals i.e., with $\sin \omega_{0} t * \sin (2 \pi \mathrm{t} / 4 \mathrm{~Tb})$. Generally, a third request butterworth filter having a cut off recurrence of $2 / 2 \mathrm{~Tb} \mathrm{~Hz}$ is utilized for the extraction of baseband information. The resultant information is gone through a sampler; A straight forward zero request sample and hold circuit is enough for this reason. 


\section{SIMULATION RESULTS AND ANALYSIS}

In simulation section, experiments have been done to find out and study the parameters that influence IEEE 802.15.4 modulation and demodulation procedure utilizing Offset Quadrature Phase Shift Key, moreoverthe outcomes are shown as previews of scope signals alsowe can know effectively what happens precisely inside an IEEE 802.15.4 transmitter. At the transmitter end, the input signal stream generated using the random integer signal generator. Input stream data rate is $250 \mathrm{Kbps}$ with $4 \mu \mathrm{s}$. Figure 4 shows the network topology of IEEE 802.15.4 system.
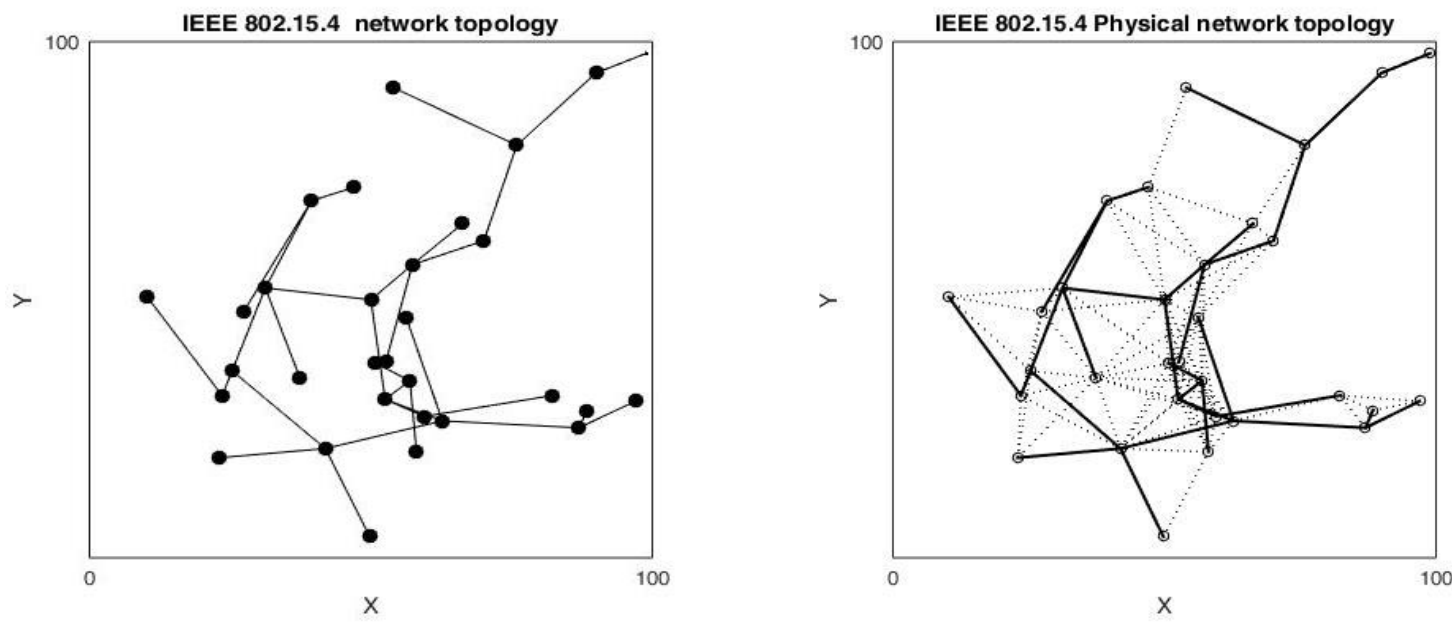

Figure 4. Simulation of network topology of IEEE 802.15.4 system

The input data is mapped into a symbolaccording to $62.5 \mathrm{~K}$ symbol per second or $16 \mu$ s symboltime period while pseudo noise sequence generator has been used to generate NP code with $2 \mathrm{Mbps}$ $(0.5 \mu \mathrm{s}$ bit time period $)$ data rate. The chip rate is equal to $8(4 \mu \mathrm{s} / 0.5 \mu \mathrm{s})$ times and $32(16 \mu \mathrm{s} / 0.5 \mu \mathrm{s})$ times the bit rate of input stream rate and symbol rate simultaneously. Furthermore, A square wave has been used as an input signal into the system, PN sequence generator has been used too. A product block has been used in the simulation to incorporate the aforementioned signals producing.

IEEE 802.15.4 needs to exploit the offset of QPSK modulation for the operation of $2.4 \mathrm{GHz}$ method. On the other hand, OQPSK has offset period equals to one-half of the pulse time frame between the quadrature pulse and in-phase pulse. This little distinction between OQPSK and QPSK will restrain the maximum extreme phase shift of a signal modulated using OQPSK to 90 degrees [23-24]. This property settles on OQPSK a better decision over QPSK for most useful implementations. According to the following Figure 5 of OQPSK modulation constellation points, each data of 2 bits is mapped into a particular signal phase.

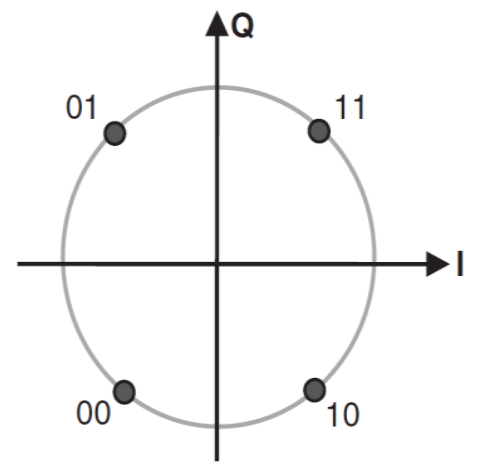

Figure 5. OQPSK Modulation constellation points 
The product output signal is fed into modulator block which uses Offset Quadrature Phase Shift Key (OQPSK) method in the transmitter section. While, the modulated signal in the transmitter side received by the receiver side then the signal demodulated by a demodulator block and generate the signal by observing these figures, we can know easily what happens exactly in-side the IEEE 802.15.4 receiver. The transmitted signal is passed through a AWGN channel [25]. The modulated signal will be received by the receiver section in the demodulator block to produce the output signal as follow. The process of the receiver section will be inversely as same as transmitter stage; by reversing process stages of the transmitter, the demodula ted signal will be processed somehow to produce the final output of the system that represented by Figure 6.
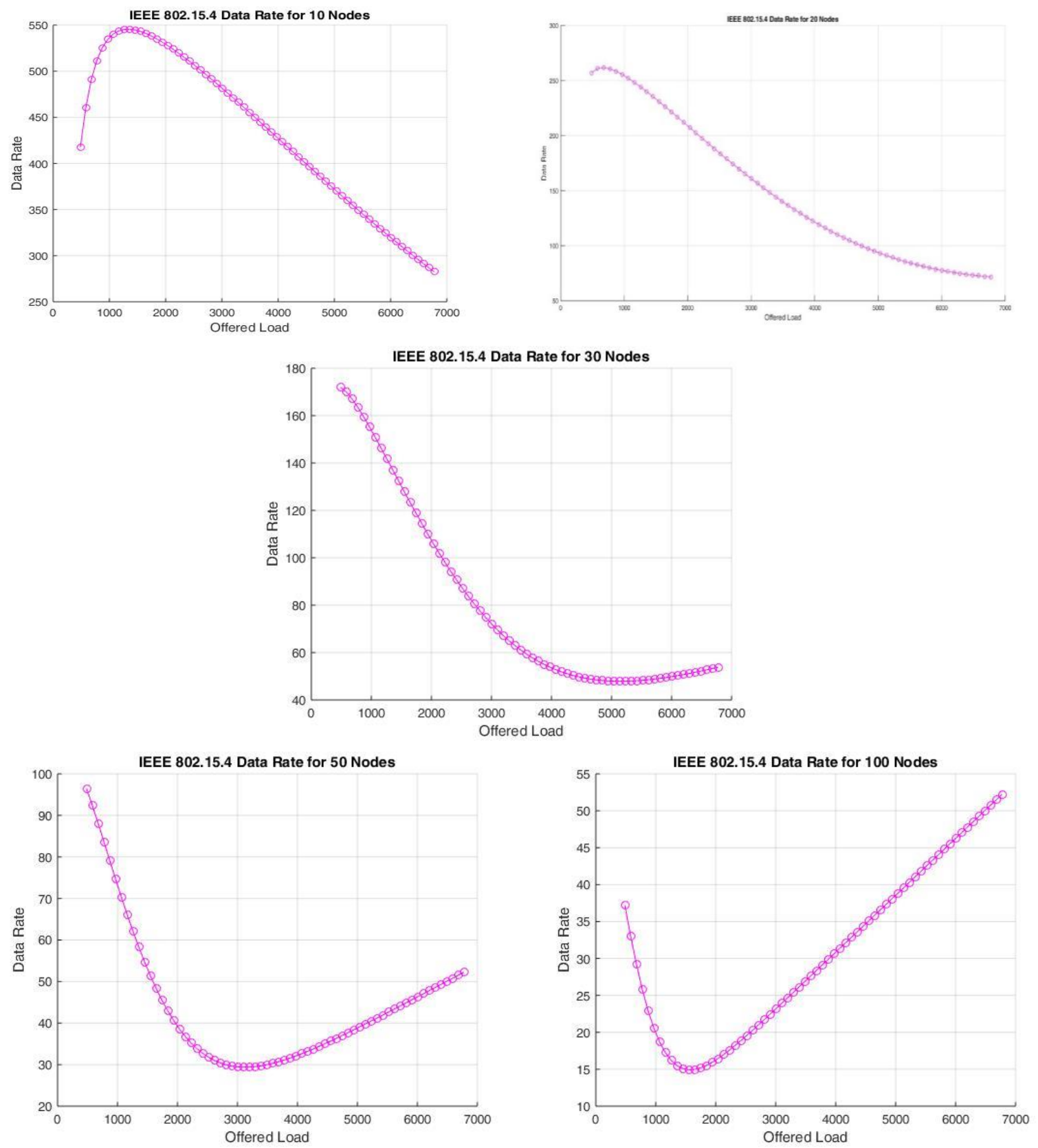

Figure 6. IEEE 802.15.4 Data rate with different number of nods

The research paper, experiments have been done to find out and study the parameters that affect IEEE 802.15.4 modulation and demodulation process using OQPSK. In experiments, we found that a synchronization between transmitter and receiver should be taken into account to modulate and demodulate the signal properly. The following results show the effects of the synchronization and DE synchronization 
system, in which, the modulation and demodulation sections have either different or same parameters. We note that there is no change in value of BER when phase shift of OQPSK in the transmitter and receiver are identical. It's clearly shown in the Figure 7 that if asymmetric parameters are used in the system, this could produce different output signals based on chosen values.

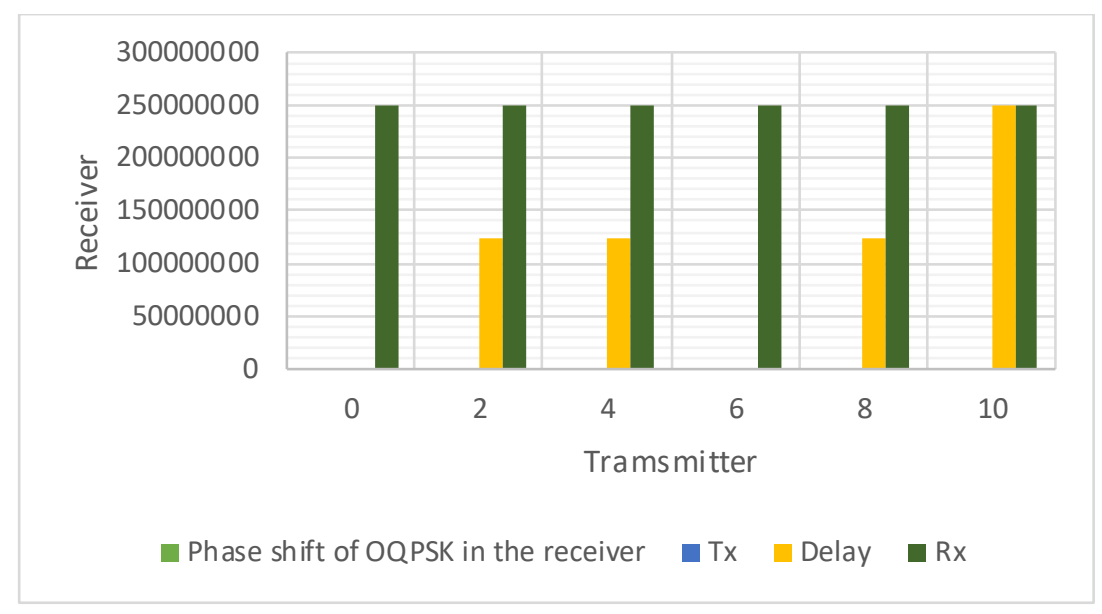

Figure 7. Phase shift of OQPSK in the IEEE 802. 15.4 recevier

\section{CONCLUSION}

The research paper has introduced a fundamental execution investigation of the IEEE 802.15.4 wireless standard through pragmatic examinations IEEE 802.15.4 is among the most conspicuous communication advancements for low power wireless systems administration, for example, wireless sensor/actuator networks (WSANs). The future work incorporates the assessment of the power utilizations, association time, a tree or a device-to-device topology, and device nodes. The research paper discussed a standard structure of IEEE 802.15.4 transmitter and receiver modulation systems by MATLAB program while they are secured and a large portion of the physical layer blocks utilized in IEEE 802.15.4 protocol. In addition, OQPSK technique is utilized in this simulation.

\section{REFERENCES}

[1] A. C. Torres, V. Ramos, T. R. Chávez and T. Flores, "Development of a network of sustainable wireless sensors through the IEEE 802.15.4 standar to determine the quality of water," 2017 IEEE 37th Central America and Panama Convention (CONCAPAN XXXVII), Managua, pp. 1-6, 2017.

[2] A. Nabila and E. B. Mohamed, "A QoS based comparative analysis of the IEEE standards 802.15.4 \& 802.15.6 in WBAN-based healthcare monitoring systems," 2019 International Conference on Wireless Technologies, Embedded and Intelligent Systems (WITS), Fez, Morocco, pp. 1-5, 2019.

[3] J. Rewienski, M. Groth, L. Kulas and K. Nyka, "Investigation of continuous wave jamming in an IEEE 802.15.4 network," 2018 22nd International Microwave and Radar Conference (MIKON), Poznan, pp. 242-246, 2018.

[4] D. Mankar and B. S. Chaudhari, "Dynamic performance analysis of IEEE 802.15.4 devices under various RF interferences," 2016 International Conference on Inventive Computation Technologies (ICICT), Coimbatore, pp. 1-4, 2016.

[5] S. Leugner and H. Hellbrück, "eNAV-Enhanced Co-Existence of IEEE 802.15.4 and IEEE 802.11," 2019 IEEE International Symposium on Local and Metropolitan Area Networks (LANMAN), Paris, France, pp. 1-6, 2019.

[6] H. Erdöl, H. Y. Özderya, C. Şişman and İ. Kaya, "Comparison of IEEE 802.15.4 and IEEE 802.15.6 standard's BER performances," 2016 24th Signal Processing and Communication Application Conference (SIU), Zonguldak, pp. 1445-1448, 2016.

[7] H. Erdöl, H. Y. Özderya, C. Şişman, T. Kayikcioglu and İ. Kaya, "BER performance comparison of IEEE 802.15.6 and IEEE 802.15.4 on PHY layer," 2016 39th International Conference on Telecommunications and Signal Processing (TSP), Vienna, pp. 108-111, 2016.

[8] F.M.Puspita, R.T.Agustin, "Bit Error Rate (BER) QoS Attribute in Solving Wireless Pricing Scheme on Single Link Multi Service Network", Int. Journal of Electrical and Computer Engineering (IJECE), vol. 8, no. 1, pp. 236-245, Feb. 2018. 
[9] C. Sum, M. Zhou, L. Lu, F. Kojima and H. Harada, "Network performance of a low energy IEEE 802.15.4/4e/4m WPAN system utilizing TV white space,"2013 IEEE 24th Annual International Symposium on Personal, Indoor, and Mobile Radio Communications (PIMRC), London, pp. 1629-1633, 2013.

[10] U. Pešović, D. Gliech, P. Planinśič, Z. Stamenkovic and S. Ranđić, "Implementation of IEEE 802.15.4 transceiver on software defined radio platform," 2012 20th Telecommunications Forum (TELFOR), Belgrade, pp. 376-379, 2012.

[11] B. Han, X. Cao and H. Shi, "Modeling the throughput of IEEE 802.15.4 based wireless networks under interference,"2017 32nd Youth Academic Annual Conference of Chinese Association of Automation (YAC), Hefei, pp. 1014-1019, 2017.

[12] Jin-Shyan Lee, "Performance evaluation of IEEE 802.15.4 for low-rate wireless personal area networks," in IEEE Transactions on Consumer Electronics, vol. 52, no. 3, pp. 742-749, Aug. 2006.

[13] E. Callaway et al., "Home networking with IEEE 802.15.4: a developing standard for low-rate wireless personal area networks," inIEEE Communications Magazine, vol. 40, no. 8, pp. 70-77, Aug. 2002.

[14] X. Ge, W. Zhang, "5G Green Mobile Communication Networks", book, Springer, Publishing House of Electronics Industry, China, pp. 4-5, 2019.

[15] E. Kadhum, R. Haitham, "Performance Analysis of IEEE 802.15.4 Transceiver System under Adaptive White Gaussian Channel", Int. Journal of Electrical and Computer Engineering (IJECE), vol. 8, no. 6, pp. 4184-4196, Dec 2018.

[16] T. Elshabrawy, "Throughput analysis of IEEE 802.15.4 enabled wireless sensor networks under WLAN interference," 2014 IEEE Fourth International Conference on Consumer Electronics Berlin (ICCE-Berlin), Berlin, pp. 467-469, 2014.

[17] A. G. Ramonet and T. Noguchi, "IEEE 802.15.4 Historical Evolution and Trends," 2019 21st International Conference on Advanced Communication Technology (ICACT), PyeongChang Kwangwoon_Do, Korea (South), pp. 351-359, 2019.

[18] F. Wunsch, H. Jakel and F. K. Jondral, "Performance Evaluation of IEEE 802.15.4 OQPSK and CSS PHY in the Presence of Interference," 2015 IEEE 82nd Vehicular Technology Conference (VTC2015-Fall), Boston, MA, pp. 1-5, 2015.

[19] S. Fang, S. Berber, A. Swain and S. U. Rehman, "A study on DSSS transceivers using OQPSK modulation by IEEE 802.15.4 in AWGN and flat Rayleigh fading channels," TENCON 2010 - 2010 IEEE Region 10 Conference, Fukuoka, pp. 1347-1351, 2010.

[20] Y. V. Varshney, A. K. Sharma, "Design \& Simulation of Zigbee Transceiver System Using Matlab, " Int. Journal of Engineering Trends and Technology (IJETT), vol.4, no.4, pp. 1316-1319, 2013.

[21] Danfeng Zhao and Tielin Zhu, "A new digital method of demodulation for MSK," 2009 4th IEEE Conference on Industrial Electronics and Applications, Xi'an, pp. 1792-1795, 2009.

[22] R. Sadr and J. K. Omura, "Generalized minimum shift-keying modulation techniques," in IEEE Transactions on Communications, vol. 36, no. 1, pp. 32-40, Jan. 1988.

[23] R. Ahmad, O. Sidek, Wan Mohd Hafizi Wan Hassin and Shukri Korakkottil Kunhi Mohd, "Implementation of IEEE 802.15.4-based OQPSK-pulse-shaping block on FPGA," 2011 IEEE International Conference on Computer Applications and Industrial Electronics (ICCAIE), Penang, pp. 459-464, 2011.

[24] S. K. Kaul, "QPSK, OQPSK, CPM Probability Of Error for AWGN and Flat Fading Channels," Wireless Communication Technologies, Spring, 2005.

[25] K. Long, V. C. Leung, H. Zhang, Z. Feng, Y. Li, and Z. Zhang, "Low-Complexity Equalization of Continuous Phase Modulation Using Message Passing," ICST Institute for Computer Sciences, Social Informatics and Telecommunications Engineering, pp. 493-505, 2018. 